\title{
Information value of magnetic resonance imaging in shunted hydrocephalus
}

\author{
Eija Pääkkö, Tuija Löppönen, Anna-Liisa Saukkonen, Juhani Pyhtinen, Juhani Laitinen, \\ Willy Serlo, Mikael Knip
}

\begin{abstract}
Objective - To study the role of magnetic resonance imaging (MRI) in evaluating children with shunted hydrocephalus.

Methods - Sixty one asymptomatic children with shunted hydrocephalus or cystic cerebrospinal fluid collections were studied by cranial MRI. The information obtained from the images was classified into three categories: provided (1) a new diagnosis, (2) additional information, or (3) no essential new information. The findings were compared with those of the last follow up computed tomograms.

Results - MRI provided a new diagnosis in seven cases $(11.5 \%)$, and additional information was obtained in $34(55 \cdot 7 \%)$ cases. In 20 cases $(32 \cdot 8 \%)$ no essential new information was obtained. MRI visualised white matter lesions and corpus callosum pathology more often than computed tomograms.

Conclusions - MRI provided new important information in cases of children with shunted hydrocephalus to such an extent that it can be recommended as the primary imaging method for every child with this disorder.

(Arch Dis Child 1994; 70: 530-535)
\end{abstract}

Although computed tomography has been used successfully for the diagnosis and follow up of children with hydrocephalus, ${ }^{1}$ small obstructive lesions may remain undetected and it has major drawbacks when attempting to illustrate the posterior fossa anatomy. Sometimes more invasive additional methods such as cisternography and ventriculography have been employed to visualise the ventricular and cisternal anatomy and cerebrospinal fluid dynamics. ${ }^{2}$ Because of its multiplanar

Table 1 Diagnosis before MRI and category in 61 patients with shunted hydrocephalus

\begin{tabular}{|c|c|c|c|c|}
\hline \multirow[b]{2}{*}{ Diagnosis before $M R I$} & \multirow[b]{2}{*}{ No (\%) } & \multicolumn{3}{|c|}{ MRI category } \\
\hline & & $\begin{array}{l}\text { Provided } \\
\text { new } \\
\text { diagnosis }\end{array}$ & $\begin{array}{l}\text { Provided } \\
\text { additional } \\
\text { information }\end{array}$ & $\begin{array}{l}\text { No essential } \\
\text { new } \\
\text { information }\end{array}$ \\
\hline $\begin{array}{l}\text { Chiari II malformation } \\
\text { Aqueductal stenosis } \\
\text { Leptomeningeal inflammation } \\
\text { Arachnoidal cyst } \\
\text { Dandy-Walker cyst or variant } \\
\text { Porencephaly } \\
\text { Vascular malformation } \\
\text { Encephalocele } \\
\text { Unknown }\end{array}$ & $\begin{array}{l}9(14 \cdot 8) \\
13(21 \cdot 3) \\
8(13 \cdot 1) \\
8(13 \cdot 2) \\
5(8 \cdot 2) \\
3(4 \cdot 9) \\
1(1 \cdot 6) \\
1(1 \cdot 6) \\
13(21 \cdot 3)\end{array}$ & $\begin{array}{l}\overline{5^{\star}} \\
1^{\star} \\
\overline{-} \\
\overline{-} \\
\overline{-} \\
1^{\star}\end{array}$ & $\begin{array}{l}9 \\
5 \\
2 \\
7 \\
5 \\
2 \\
1 \\
1 \\
2\end{array}$ & $\begin{array}{l}\overline{3} \\
5 \\
1 \\
\overline{1} \\
\overline{-} \\
\overline{0}\end{array}$ \\
\hline Total No (\%) & $61(100)$ & $7(11 \cdot 5)$ & $34(55 \cdot 7)$ & $20(32 \cdot 8)$ \\
\hline
\end{tabular}

^New diagnoses were Chiari I malformations. imaging capacity and superiority in imaging the posterior fossa, magnetic resonance imaging (MRI) is nowadays the method of choice for many paediatric brain conditions. $^{3-6}$ It is more sensitive to small structural changes contributing to the development of hydrocephalus ${ }^{7-9}$ and to anatomical changes caused by distended ventricles. ${ }^{10}$ We report here on 61 asymptomatic children with shunted hydrocephalus who were evaluated by MRI to assess the value of new information that can be obtained by this technique.

\section{Subjects and methods}

Sixty one children, 33 boys and 28 girls (age range $5 \cdot 0-19.8$ years, median 13.0 years), provided with a shunt for hydrocephalus or cystic cerebrospinal fluid collections were studied by cranial MRI. This series represents part of a larger survey of growth and physical maturation in hydrocephalic children. Children with brain tumours, achondroplasia, and moderate or severe mental retardation were excluded. The patients had undergone the first shunting procedure at a median age of 0.6 years (range $0-14.2$ years), and the interval from the first operation to MRI varied from 1.3 to 19.7 years (median 10.5 years). The diagnoses before MRI are shown in table 1. Fourteen of the patients $(23 \%)$ had had one or two shunt infections and $28(46 \%)$ one or more revisions of the proximal end of the shunting catheter. Subdural fluid collections were documented in nine patients. Preoperative computed tomograms were available in 39 cases. Fourteen patients with hydrocephalus diagnosed in the 1970s had undergone pneumoencephalography (PEG), and ventriculography and cisternography had been performed in two cases each. Follow up computed tomograms were available for all 61 patients. The time interval from the last computed tomogram to $M R I$ ranged from 0 to 3.6 years (median $1 \cdot 1$ years).

MRI was performed with a $1.0 \mathrm{~T}$ superconducting unit (Magnetom, Siemens). Sagittal and precontrast and postcontrast coronal $\mathrm{T} 1$ weighted (TR 500-690 ms, TE $15 \mathrm{~ms}$, two excitations) images were obtained with a slice thickness of 3 and $5 \mathrm{~mm}$, respectively. In seven cases the sagittal images were $5 \mathrm{~mm}$ thick and no postcontrast study was done. T2 weighted (TR $2300 \mathrm{~ms}$, TE 15-90 ms, one excitation) images were obtained in the axial plane with a slice thickness of $5 \mathrm{~mm}$ in all cases. The images were analysed by two 
Table 2 MRI findings compared with computed tomography in 61 patients with shunted hydrocephalus; figures are number (\%)

\begin{tabular}{lll}
\hline Finding & $\begin{array}{c}\text { Computed } \\
\text { tomography }\end{array}$ & MRI \\
\hline Focal white matter changes & $1(1 \cdot 6)$ & $20(32 \cdot 8)^{\star}$ \\
Calcifications & $3(4 \cdot 9)$ & $0(1$ increased \\
& & signal $)$ \\
Heterotopia & $2(3 \cdot 3)$ & $3(4 \cdot 9)$ \\
Microgyria & $\mathrm{NA}$ & $6(9 \cdot 8)$ \\
Corpus callosum abnormality & $16(26 \cdot 2)$ & $37(60 \cdot 7)^{\star}$ \\
Meningeal enhancement & $\mathrm{NA}$ & $17(31 \cdot 5) \dagger$ \\
Aqueduct open & $\mathrm{NA}$ & $17(27 \cdot 9)$ \\
\hline
\end{tabular}

NA: not appreciable.

${ }^{\star} \mathrm{p}<0 \cdot 001$. + Fifty four patients had a postcontrast study.

radiologists, paying special attention to posterior fossa structures, patency of the aqueduct, white matter lesions, migrational disorders, size and shape of the corpus callosum and enhancement of the cranial meninges. Preoperative computed tomography, PEG, ventriculography, and cisternography were reviewed, as were the last follow up computed tomograms. The information obtained from MRI was classified into three
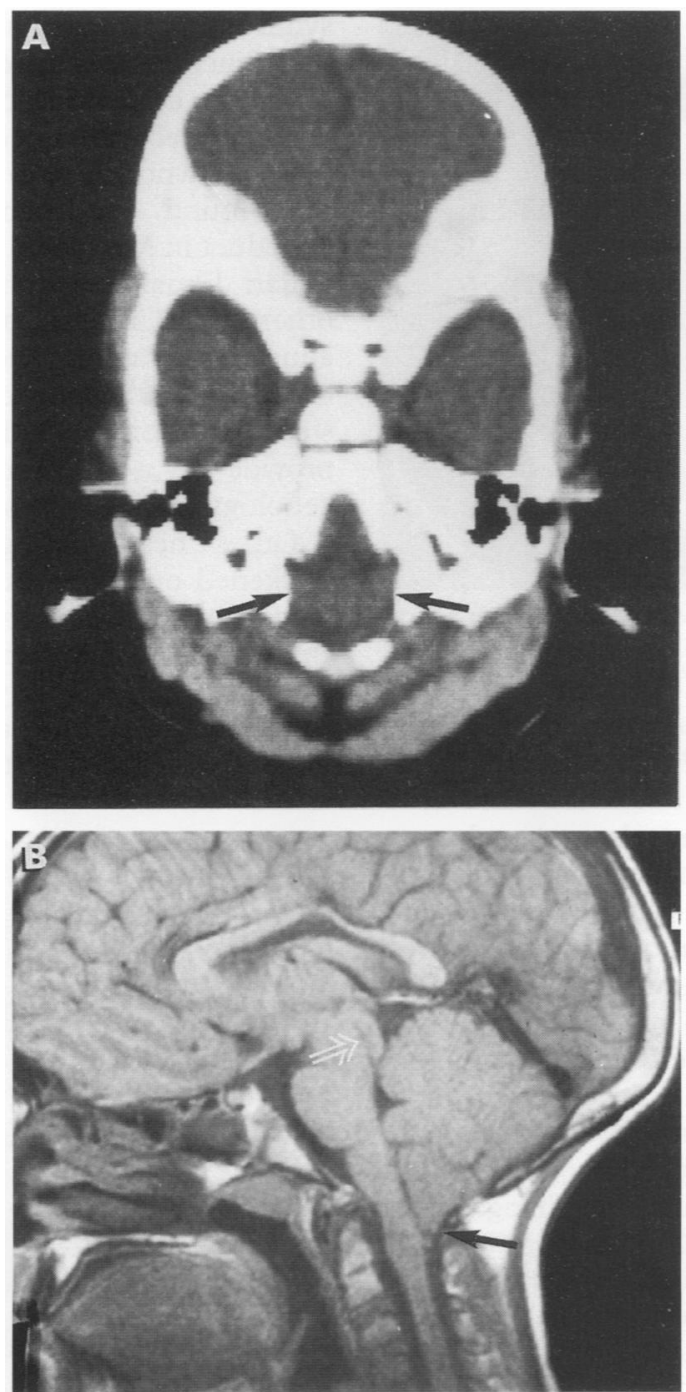

Figure 1 Chiari I malformation in a patient with an original diagnosis of aqueductal stenosis. Computed tomogram below the level of the foramen magnum $(A)$ shows the low position of the cerebellar tonsils (arrows), which was appreciated only retrospectively. The sagittal T1 weighted MRI scan (B) visualises clearly the cerebellar tonsils below the level of the first cervical vertebra (black arrow). The aqueduct (open arrow) is not open, but the slit-like ventricles exclude reliable evaluation of its patency.

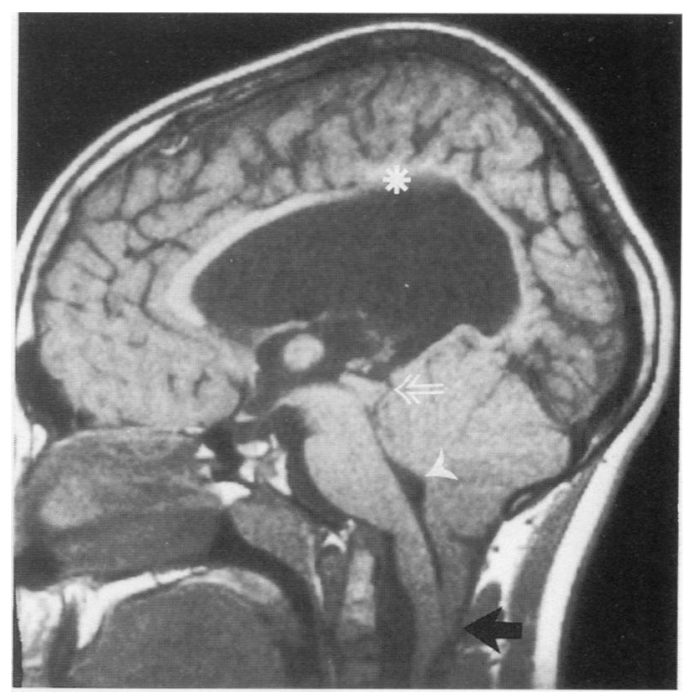

Figure $2 T 1$ weighted sagittal MRI scan of a patient with a Chiari II malformation visualises well the low position of the inferior cerebellum (black arrow) and the fourth ventricle (arrowhead). Other typical features are tectal beaking (open arrow) and dysgenesia of the corpus callosum (asterisk). The lateral ventricle is enlarged.

categories: provided (1) a new diagnosis, (2) additional information, or (3) no essential new information.

Six patients needed sedation, usually induced with an intravenous injection of thiopentone, during the examination. Their cardiac and respiratory status was monitored and a physician was present in the room during the examination.

The data were analysed statistically using Fisher's exact probability test. Probability values less than 0.05 were considered significant.

The research was approved by the ethical committee of the medical faculty, and informed consent was obtained from the patients and their parents.

\section{Results}

The distribution of the series into MRI categories is shown in table 1 and comparison of the findings with those observed by computed tomography is presented in table 2 . MRI provided a new diagnosis in seven cases $(11.5 \%)$, all new diagnoses being Chiari I malformations (table 1). Retrospectively assessed, one of the new Chiari I malformations could have been suspected from the computed tomograms (fig 1), while in others, the images did not reach low enough to visualise the low positioned cerebellar tonsils. Additional information was obtained in $55.7 \%$ of cases (table 1). MRI showed the structural changes associated with Chiari II malformation (fig 2), arachnoidal cyst, Dandy-Walker cyst or variant (fig 3), and porencephaly extremely clearly. The patient with an operated occipital encephalocele had cerebellar hemispheres that were mere 'raisins' and smaller than previously suspected (fig 4). Unsuspected findings of white matter changes, corpus callosum abnormalities, and migrational disorders were also considered important additional information. 

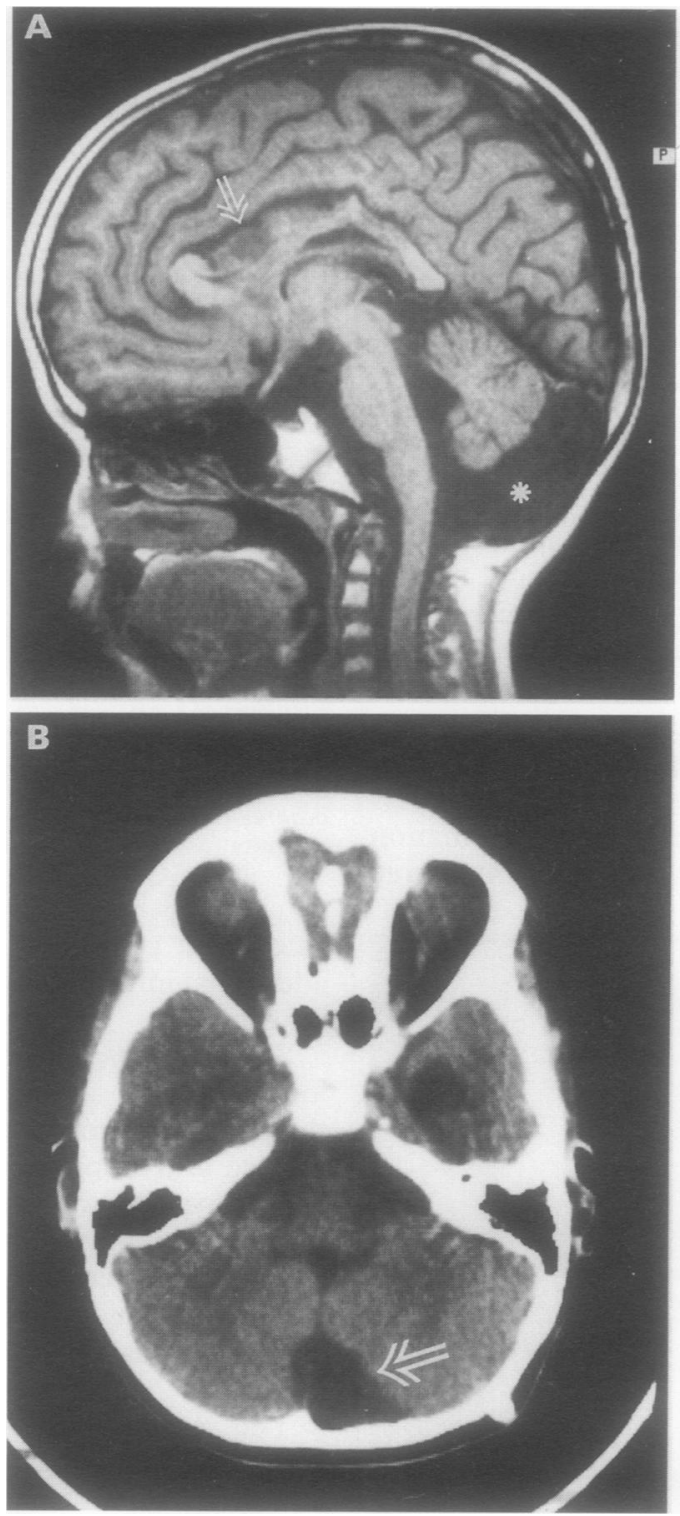

Figure $3 T 1$ weighted sagittal MRI scan $(A)$ in a patient with the Dandy-Walker variant reveals a larger posterior foss a cerebrospinal fluid space (asterisk) than is appreciable in a computed tomogram (B) 1.5 years earlier (open arrow). MRI also shows irregularity and an abnormally low signal in the corpus callosum (open arrow).

Computed tomography showed only one case with focal white matter hypodensity as opposed to 20 cases seen as an increased signal on T2 weighted MRI, but it did visualise focal parenchymal calcifications in three cases which were not seen in MRI (table 2). Only in one case was a paradoxical faint increase in signal intensity in the $\mathrm{T} 1$ weighted images observed in corresponding areas. Heterotopic grey matter foci were visualised almost equally well on computed tomography, although the changes were less extensive than in MRI. Microgyria was not appreciable on the computed tomograms.

The corpus callosum was visualised extremely well in sagittal MRI, and in 12 cases $(19.7 \%)$ it was attenuated and elevated due to large ventricles. Other findings were hypoplasia/atrophy $(n=4)$, mild $(n=8)$ or severe $(n=10)$ dysgenesia, and tissue damage with signal changes (low in $\mathrm{T} 1$ weighted images and high in T2 weighted ones) $(n=3)$ (fig 3$)$. Computed tomography showed only the most severe changes.
When evaluating the 54 patients with postcontrast MRI studies, 14 out of 17 patients $(82 \%)$ with abnormal enhancement of the cranial meninges (fig 5) had had predisposing factors such as shunt infections, one or more revisions of the proximal end of the catheter, or subdural effusions or haematomas compared with 17 out of $37(46 \%)$ without enhancement. The difference between the groups was significant $(p=0.01)$. Of the predisposing factors one or more revisions of the proximal end of the catheter was seen more often $(p=0.04)$ in the group with enhancing meninges $(12 / 17$ with enhancement and 15/37 without enhancement), whereas such difference was not seen with shunt infections and subdural fluid collections.

In the whole series the aqueduct was unequivocally open in only 17 cases $(27 \cdot 9 \%)$, all of whom had a normal or increased ventricular size. An obliterated or equivocal aqueduct was associated with slit ventricles in 21 out of 35 cases $(60 \%)$ when the patients with a Chiari II malformation were excluded (aqueductal stenosis is a characteristic of the syndrome). A signal void, indicating flow in the aqueduct, was seen in seven cases (five open, two equivocal). None of the five patients with suspected aqueductal stenosis before MRI, in whom a new Chiari I malformation was found, had an unequivocally open aqueduct in MRI, and aqueductal stenosis and Chiari I malformation may coexist. On the other hand, in two patients with presumed aqueductal stenosis the aqueducts were open on MRI making the diagnosis of aqueductal stenosis dubious. MRI did not, however, provide any new information in respect to the aetiology in these cases.

Other new findings on MRI included thickened optic nerves and chiasma $(n=1)$, a high, convex pituitary gland touching the chiasma $(n=2)$, prominent meningeal vessels (raising the possibility of an arteriovenous fistula) $(n=1)$, and a possible septa in a shunted arachnoid cyst $(n=1)$. These findings

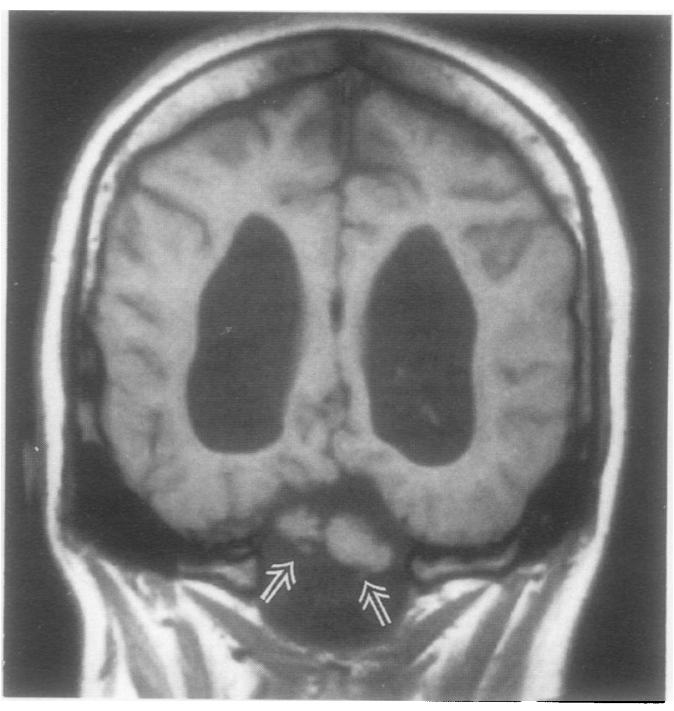

Figure 4 Coronal T1 weighted MRI scan of a patient with an operated occipital encephalocele shows extreme hypoplasia of the cerebellar hemispheres (open arrows). The lateral ventricles are enlarged. 


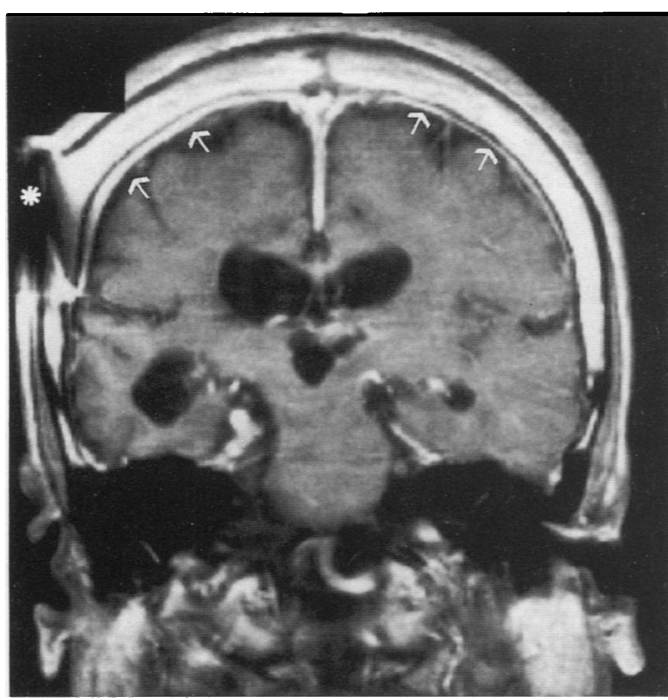

Figure 5 Coronal postcontrast T1 weighted MRI scan reveals extensive enhancement in the cranial meninges (arrows) of a patient with an operated arteriovenous malformation. He had also had postoperative subdural effusions. The skull bone is thick. The low signal area on the right (asterisk) is due to metal parts in the shunt.

on MRI resulted in additional examinations to explore their aetiology.

\section{Discussion}

MRI provided a new diagnosis in close to $12 \%$ of the present series of 61 asymptomatic patients with hydrocephalus. This observation is not only of academic interest but has major implications for the follow up of these patients. All the new diagnoses were Chiari I malformations, and it is known that syringomyelia may develop in the syndrome. In the sagittal $\mathrm{T} 1$ weighted brain scans none of our patients showed evidence of syringomyelia in the upper cervical cord, but MRI of the spine was scheduled in three cases. It is always difficult for the clinician to differentiate whether the patients' symptoms are due to the disease itself or are iatrogenic (for example, slit ventricles, repeated operations, etc). When MRI reveals developmental anomalies some of the symptoms may be better understood, and unnecessary anxiety on the part of the patients, their parents, and the doctor can be avoided.

Chiari I malformation may be diagnosed on computed tomograms from low positioning of the cerebellar tonsils and fourth ventricle, ${ }^{1}$ as was illustrated retrospectively in one of our cases (fig 1). The resolution of computed tomography in respect to the tonsils may not be good enough, however, and especially if the first scan does not reach low enough, the malformation may remain undetected. Follow up computed tomograms are usually restricted to the upper ventricular area in order to document any change in the size of the ventricles and are accordingly not diagnostically appropriate with respect to the posterior fossa.

The new Chiari I malformations in our cases may nevertheless be a secondary finding and not necessarily the cause of hydrocephalus. Chiari I malformation may coexist with aqueductal stenosis and the aqueductal stenosis is then the likely determinant of hydrocephalus.
Shunting may affect the position of the cerebellar tonsils and a slightly low position of the tonsils may be seen in normal population. The clinical findings in Chiari I malformation usually manifest first in early to middle adulthood, ${ }^{7}$ which makes it less likely in the paediatric population we studied.

Separating Chiari I and Chiari II malformations was not always straightforward, although a Chiari II malformation is almost invariably associated with meningomyelocele. In three of the new cases with a Chiari I malformation other significant findings, including severe corpus callosum changes, wide tentorial incisura, upward bulging of vermis and cerebellum, gaping foramen magnum, enlarged interhemispheric fissure, and other wide posterior subarachnoidal cerebrospinal fluid spaces were seen. These findings resembled those seen in Chiari II malformations. We mostly relied on the posterior fossa changes, which were more extensive in Chiari II malformation with downward displacement of the fourth ventricle, medulla and cerebellum, whereas in Chiari I malformation only the cerebellar tonsils were seen below the foramen magnum. The other morphological changes in these three patients with Chiari I malformation may have been the sequelae of a colpocephalic hydrocephalus seen in the preshunting computed tomograms.

MRI provided additional information in a substantial proportion of our patients $(55 \cdot 7 \%)$. Many major parenchymal changes (for example, white matter lesions, microgyria, and corpus callosum abnormalities) were depicted more distinctly than in computed tomography, which is not surprising, as the superiority of MRI is well documented in these instances. ${ }^{41112}$ The time elapsing since computed tomography was in some cases quite long, however (up to 3.6 years), which may have influenced the results, especially the detection of white matter changes. In three cases parenchymal calcifications were present on computed tomography that were not visible on MRI (only one showed a faint increase in signal intensity). This is a known defect in MRI that may even influence the classification of hydrocephalus, as these calcifications are often associated with previous infection and may suggest leptomeningeal inflammation as the cause.

Meningeal enhancement was seen more often in patients with one or more revisions of the proximal end of the catheter than in those without revisions. Meningeal enhancement in MRI may involve multiple aetiological factors, including postoperative, infectious, or carcinomatous inflammation and fibrosis. ${ }^{13-16}$ Accordingly, meningeal enhancement is not an alarming finding in shunted patients but probably reflects only the sequela of many operations, effusions, and infections.

Cysts and other cerebrospinal fluid spaces were also better delineated, due to the multiplanar imaging. We encountered, however, difficulties in diagnosing and categorising posterior fossa cerebrospinal fluid collections, Dandy-Walker cyst, Dandy-Walker variant, and mega cisterna magna, on MRI. After 
shunting the appearance of the fluid collections may alter and the lesions may be obvious only in the preshunting studies. ${ }^{17} \mathrm{MRI}$ has also provided new information on these collections which may not be separate entities, but represent a continuum of developmental anomalies of the posterior fossa. ${ }^{18} \mathrm{~A}$ new term, DandyWalker complex, has been suggested to describe these changes. ${ }^{18}$

Evaluation of the aqueduct proved more complicated than expected, and only $27.9 \%$ of the conduits were found to be unequivocally open. In normal subjects without hydrocephalus or shunting the aqueduct is always well visualised in sagittal T1 weighted images. ${ }^{8}$ Shunting, and especially slit ventricles, will affect the size of the small aperture and prevent any reliable diagnosis of stenosis. This implies that the diagnosis of aqueductal stenosis must be made before the shunting procedure if MRI is to be used. A flow related signal void was not of much help as it was seen in only seven cases, whereas in normal children it may be seen in up to $100 \% .{ }^{19}$ Flow compensation techniques are nowadays used routinely in MRI to reduce flow related artifacts, but this may at the same time abolish the useful signal void indicating flowing cerebrospinal fluid, ${ }^{20}$ and shunting itself may affect the flow void. Gradient echo cine MRI and phase contrast velocity MRI may provide better detection of flowing cerebrospinal fluid, and could be used as an additional method in obstructive and communicating hydrocephalus. ${ }^{21} 22$

The observation that MRI clarified only one of 13 cases with unknown aetiology was disappointing, especially as it offered additional information only in two of these subjects. The MRI method used here could not visualise aqueductal stenosis reliably, nor was it able to suggest any defect in the resorption of cerebrospinal fluid.

In conclusion, MRI provided new important information in cases of children with shunted hydrocephalus to such an extent that it can be recommended as the primary imaging method for every child with this disorder whenever possible. To make the most of it for defining the aetiology of hydrocephalus it should initially be performed before the first operation. The limited availability of the technology and difficulties in patient monitoring, as well as the need for sedation in the cases of small or retarded children may nevertheless reduce the feasibility of MRI. MRI could also be used in the future instead of computed tomography for monitoring ventricular size. The number of imaging planes and sequences can be reduced in order to cut down the imaging time needed. No substantial information would be lost by using only a T2 weighted axial MRI scan, and more information would be obtained than with computed tomography. This research was supported by the Sigrid Juselius Foundation,
Helsinki (TL, MK), the Medical Research Council of the Academy of Finland (EP) and the Alma and K A Snellman Academy of Finland (EP) and the A
Foundation, Oulu, Finland (EP, TL).

1 Naidich TP, Epstein F, Lin JP, Kricheff II, Hochwald GM Evaluation of pediatric hydrocephalus by computed tomography. Radiology 1976; 119: 337-45.
2 Naidich TP, Schott LH, Baron RL. Computed tomography in evaluation of hydrocephalus. Radiol Clin North Am 1982; 20: 143-67.

3 Packer RJ, Zimmerman RA, Bilanuik LT, et al. Magnetic resonance imaging of lesions of the posterior fossa and upper cervical cord in children. Pediatrics 1985; 76: upper

4 Nowell MA, Grossman RI, Hackney DB, Zimmerman RA, Goldberg HI, Bilanuik LT. MR imaging of white matter disease in children. $A \mathcal{F} N R$ 1988; 9: 503-9.

5 Knaap van der MS, Valk J. Classification of congenital abnormalities of the CNS. AFNR 1988; 9: 315-26.

6 Wolpert SM, Anderson M, Scott RM, Kwan ESK, Runge VM. Chiari II malformation: MR imaging evaluation. AFNR 1987; 8: 783-92.

7 Barkovich AJ, Wippold FJ, Sherman JL, Citrin CM. Significance of cerebellar tonsillar position on MR. AfNR 1986; 7: 795-9.

8 Kemp SS, Zimmerman RA, Bilanuik LT, Hackney DB, Goldberg HI, Grossman RI. Magnetic resonance imaging of the cerebral aqueduct. Neuroradiology 1987; 29: 430-6. 9 Altman NR, Naidich TP, Braffman BH. Posterior fossa malformations. AfNR 1992; 13: 691-724.

10 El Gammal T, Allen MB Jr, Brooks BS, Mark EK. MR evaluation of hydrocephalus. AfNR 1987; 8: 591-7.

11 Barkovich AJ, Norman D. Anomalies of the corpus callosum: correlation with further anomalies of the brain. AfNR 1988; 9: 493-501.

12 Osborn RE, Byrd SE, Naidich TP, Bohan TP, Friedman H. MR imaging of neuronal migrational disorders. AfNR 1988; 9: 1101-6.

13 Destian S, Heier LA, Zimmerman RD, Morgello S, Deck MDF. Differentiation between meningeal fibrosis and chronic subdural hematoma after ventricular shunting: value of enhanced CT and MR scans. AfNR 1989; 10: 1021-6.

14 Pääkkö E, Patronas NJ, Schellinger D. Meningeal GdDTPA enhancement in patients with malignancies. f Comput Assist Tomogr 1990; 14: 542-6.

15 Phillips ME, Ryals TJ, Kambhu SA, Yuh WTC. Neoplastic vs inflammatory meningeal enhancement with GdDTPA. F Comput Assist Tomogr 1990; 14: 536-41.

16 Hudgins PA, Davis PC, Hoffman JC Jr. Gadopentate dimeglumine-enhanced MR imaging in children following surgery for brain tumor: spectrum of meningeal findings. surgery for brain tumor:

17 Wolpert SM, Scott RM, Runge VM, Kwan ESK. Difficulties in diagnosing posterior fossa fluid collections after shunting procedures. AFNR 1987; 8: 653-6

18 Barkovich AJ, Kjos BO, Norman D, Edwards MS. Revised classification of posterior fossa cysts and cystlike malformations based on the results of multiplanar MR imaging. $A 7 N R$ 1989; 10: 977-88.

19 Hayakawa K, Konishi Y, Kuriyama M, Konishi K, Matsuda T. Cerebrospinal fluid flow void in children. Neuroradiology 1993; 35: 443-6.

20 Bradley WG Jr, Whittemore AR, Kortman KE, et al. Marked cerebrospinal fluid void: indicator of successful Marked cerebrospinal fluid void: indicator of successful shunt in patients with suspected normal
cephalus. Radiology 1991; 178: 459-66.

21 Nitz WR, Bradley WG Jr, Watanabe AS, et al. Flow dynamics of cerebrospinal fluid: assessment with phase-contrast velocity MR imaging performed with retrospective cardiac gating. Radiology 1992; 183: 395-405.

22 Quencer RM. Intracranial CSF flow in pediatric hydrocephalus: evaluation with cine-MR imaging. AfNR 1992; 13: $601-8$.

\section{Commentary}

Pääkkö et al have reported new and interesting observations about the MRI scan of shunted hydrocephalic children. Their conclusion has important management implications for these children - mainly that the clinician should use MRI for the evaluation of hydrocephalic patients in preference to computed tomography. They cite the superiority of MRI in providing a new diagnosis, as well as providing additional imaging information when the two are compared.

The additional information that the MRI provided was not altogether unexpected, partly because of the nature of the MRI process and partly because of the more extensive anatomical screening it provides, particularly of the posterior fossa. They report additional abnormalities such as defects in the corpus callosum and ectopic grey matter, abnormalities of the gyri, posterior fossa anatomy, optic nerves, chiasm and pituitary, and the not previously recognised meningeal enhancement in these 